\title{
Data reduction for mixed integer linear programming in complex energy systems
}

\author{
Luise Middelhauve $^{a}$, Natalija Ljubic ${ }^{a}$, Julia Granacher ${ }^{a}$, Luc Girardin $^{a}$, and François Maréchal ${ }^{a}$ \\ a Industrial Processes and Energy Systems Engineering, École Polytechnique Fédérale de Lausanne, Sion, \\ Switzerland, luise.middelhauve@epfl.ch
}

\begin{abstract}
:
The task of optimally designing and scheduling energy systems with a high share of renewable energies is complex and computationally demanding. A widespread method for tackling this task is to apply mixed integer linear programming (MILP). Even though the branch-and-bound algorithms used for solving these programs have seen significant improvements in the last years, many problems cannot be solved without further time series aggregation (TSA) methods.

State of the art approaches tackle TSA by using well known machine learning techniques to cluster yearly input data to typical periods. However, latter algorithms are usually evaluated by indicators on the performance of the algorithms themselves rather than the MILP optimization model. Furthermore, the selection of the optimal number of typical periods is commonly a subjective imposition of thresholds on these performance indicators. The issue of computational effort is eased by this generally accepted algorithm, but is still limited by the size of the problem, especially the number of integer decisions.

This paper aims at proposing a algorithm for systematically reducing the input data for MILP optimization models and choosing the appropriate size. Contrary to most existing studies, the focus is on the impact on the objective function as well as the integer decision rather than on the quality of the clustering algorithm. The subject is addressed by exploiting the two-stage character of optimal design and scheduling of the system by sequentially performing k-medoids clustering. The demonstration of the algorithm on two case studies shows that a few typical periods are sufficient to achieve near optimal decisions. Multi objective optimization (MOO) is performed to assess the quality of the data reduction. The proposed approach is outperforming state of the art algorithms for TSA by reducing CPU time of more than $40 \%$. The case study furthermore reveals that the runtime of the MOO can be reduced by approximately $90 \%$ while diverting less than $2 \%$ on Pareto optimal solutions.
\end{abstract}

\section{Keywords:}

Optimal Scheduling and Design, Renewable energies, Building energy systems, Mixed integer linear programming, kmedoids clustering, Dimensional Reduction

\section{Introduction}

The energy sector is currently facing multiple transforming forces. In context of rising concerns for anthropogenic $\mathrm{CO} 2$ emissions, the integration of renewable energy sources is becoming an increasingly widespread solution for reducing the carbon footprint of energy systems [1]. Decentralization and the increasing volatility in energy generation highlight the importance of strategically planning the interaction between building energy systems and the electricity power grid. The optimal integration of renewable resources in energy systems is a complex task as both aspects, optimal scheduling of operation as well as strategic planning of system design have to be considered side by side. Energy system optimization using Mixed-Integer Linear Programming (MILP) where an integer represents the decision for or against installing a technology, is a widely adapted framework. The mismatch between non-dispatchable renewable energy sources and typical demand profiles additionally require the consideration of time series with resolutions of several minutes to maximal one hour. The challenge of the simultaneous inclusion of integer decisions, a project horizon of several decades as well as hourly time series result in not only the need of a large amount of data but also to computational extensive problems. To make the problem computationally tractable, simplifications can be either made in the spatiotemporal, that is reducing input data, or techno-economic dimension, corresponding to reducing complexity of component modelling [2]. This work focuses on the spatio-temporal reduction. The state of the art is briefly discussed in the following section.

\subsection{State of the art}

The aggregation of time series to typical periods is specifically popular, as patterns occur naturally in the supply and demand of energy, which arise in the time dimension through hourly, daily and seasonal cycles. A recent comprehensive review by Hoffman et al. [2] analyze Time Series Aggregation (TSA) methods for modeling energy systems applied in 130 different publications. Schütz et al. [3] compare different aggregation methods for the selection of typical demand days, such as k-means, k-medians, k-centers, k-medoids, seasonal and monthly based classification. Both studies [2,3] show that the more intuitive aggregation methods of seasons 
or months lead to significantly larger errors than the machine learning methods for the same computation time. Algorithms within the latter category performed similarly well, although k-medoids were most reliable for approximating costs. Schütz et al. [3] show that the typically applied assessment of the aggregation by using Sum of Squared Errors (SSE) is not sufficiently suitable for energy system synthesis problems.

State of the art approaches in modelling complex energy systems almost exclusively apply performance indicators of the algorithm themselves such as SSE, Root Mean Square Deviation (RMSD), Mean Average Percentage Error (MAPE) or silhouette index for defining the length and amount of typical periods [4-6]. This algorithm does not provide sufficient guarantee of aggregation quality with respect to the optimal objective value and unit choices. Therefore, Brodrick et al. [7] consider the change of unit decision while increasing the amount of typical periods next to the SSE] of the k-means algorithm. Bahl et al. [8] developed a systematic method for bounding the error of the aggregation in the objective function. This has laid the foundation for a rigorous formulation of time series relaxation and aggregation comprising a method for effectively including seasonal storage [9, 10]. The mathematically rigorous formulation [9] creates an upper and lower bound of the objective function and iterates the procedure by increasing the resolution of TSA until an optimally gap criterion is met. The lower bound is found by solving a relaxed problem, whereas the upper bound is generated by solving the Operating Problem (OP) with the full time series on predefined unit sizes provided by the solution of the aggregated input data [9].

\subsection{Gaps and contribution}

As previous literature review reveals, machine learning algorithms for data reduction techniques are not very well integrated in the optimization technique of complex energy systems. Thus, this paper proposes a new iterative procedure of TSA for complex energy systems by evaluating the clustering on the objective function as well as on integer variables.

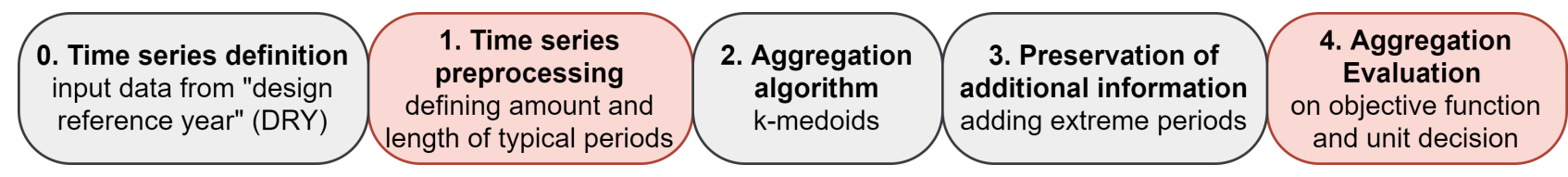

Figure 1: Procedure of time series aggregation with relevant steps highlighted in red

Figure 1 gives an overview of the general steps involved in TSA, specifying which approach is used in this paper and highlighting the steps that are varied and analyzed. In previous section, $\mathrm{k}$ - medoids algorithm is identified as most reliable for economic evaluation of energy systems. Hence, k-medoids algorithm is chosen as aggregation method in step 2 (compare Figure 1 ).

\section{Materials and methods}

The aim of this work is to bridge the gap between well established machine learning techniques and the optimization framework of complex energy systems. Therefore, the iterative procedure of reducing data is described at first (Section 2.1.), before the application case of a building energy model is introduced (Section 2.2.).

\subsection{Time series aggregation (TSA)}

An overview of the proposed algorithm is displayed in Figure 2. It consists of two parts, of which the first focuses on the objective function evaluation, whereas the second evaluates the integer decision variables. Furthermore, it defines not one solution for the choice of unit design, but a list of solutions named as the "solution space".

\subsubsection{Algorithm part 1}

The first part is characterized by an iterative process with two separate convergence criteria on the value of the objective function. With every iteration the percentage change between the solution of the Aggregated Problem (AP) based on k-medoid clustering between $\mathrm{n}$ and $\mathrm{n}-1$ clusters is calculated. Only when the first convergence criterion, $\left|\epsilon_{a}\right| \geq \frac{A P_{n}-A P_{n-1}}{A P_{n}}$, is met, the $\mathrm{OP}$ on the full time series but with fixed unit sizes is solved. Then the second convergence criterion $\left|\epsilon_{b}\right| \geq \frac{O P_{n}-A P_{n}}{A P_{n}}$ is evaluated and the iteration continues with the calculation of both problems until the second criterion is met. Then finally, the sizing and selection variables are added as a result to the solution space.

\subsubsection{Algorithm part 2}

The second part is introduced after arriving at $\mathrm{n}$ clusters which provide the first element in the solution space. It consists of recalculating the $\mathrm{AP}$ for another $\mathrm{m}$ steps with increasing amounts of typical periods. The purpose 


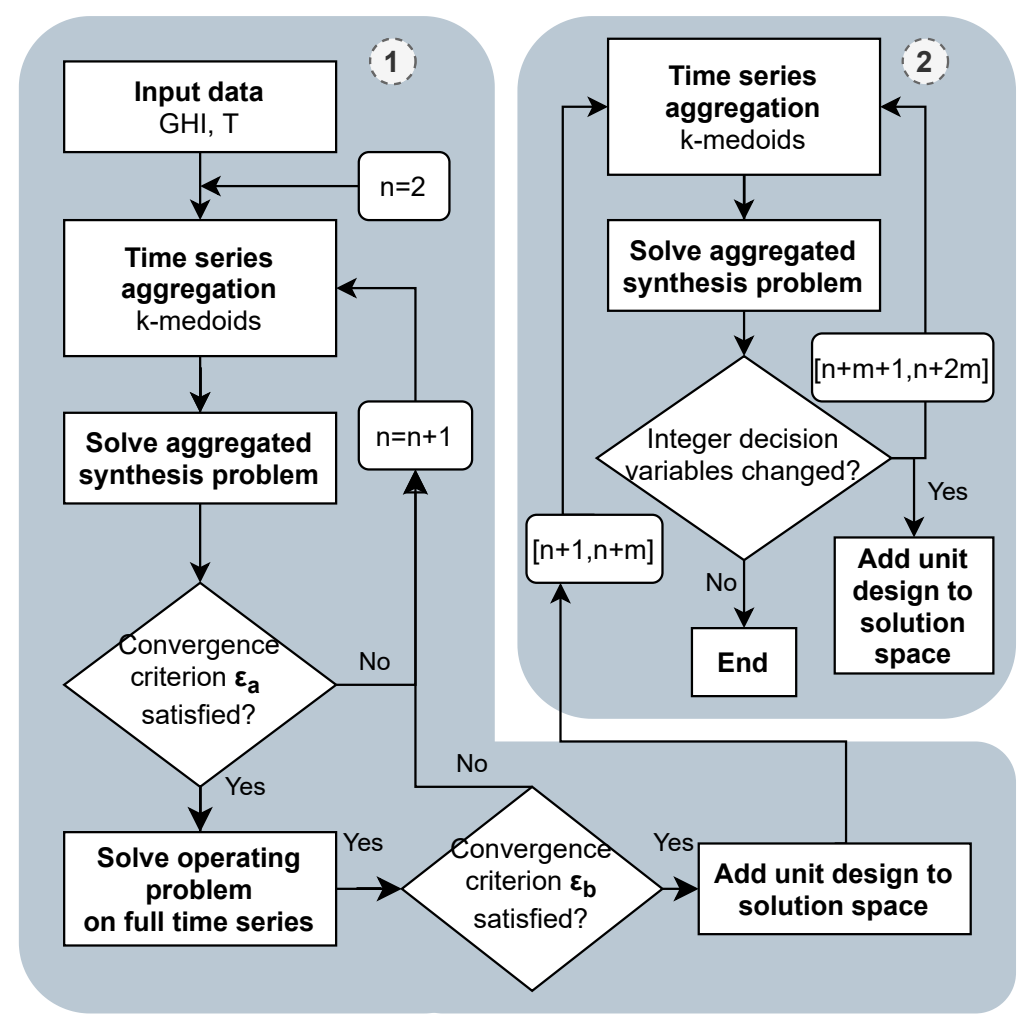

Figure 2: Overview of the proposed iterative procedure for systematic input data reduction

is to compare the integer decisions and possibly add new elements to the solution space that provide different unit choices, but have a similar value of the objective function. Depending on the type of energy system and the amount of possibilities available for unit decisions, the integer variables might remain still the same after $\mathrm{n}+\mathrm{m}$ iterations. In this case, the procedure stops with $\mathrm{n}+1$ typical periods.

\subsubsection{Tuning parameter}

The following list details the definition and a possible choice of tuning parameters:

- Convergence criterion $\left|\epsilon_{a}\right| \geq \frac{A P_{n}-A P_{n-1}}{A P_{n}}$ : Variation between the solution of the aggregated problem between $\mathrm{n}$ and $\mathrm{n}-1$ clusters; $\epsilon_{a}=5 \%$.

- Convergence criterion $\left|\epsilon_{b}\right| \geq \frac{O P_{n}-A P_{n}}{A P_{n}}$ : Variation between the solution of the aggregated problem of $n$ clusters and the non-aggregated operating problem with fixed unit decisions from the aggregated synthesis; $\epsilon_{b}=5 \%$.

- Amount of additional iterations comparing unit decisions $m$ : The amount of additional aggregated synthesis solutions that are tested for; $m=3$.

The tuning choice of $m$ specifically can be made depending on the problem complexity and amount of possible integer decisions that could provide similar results.

\subsubsection{Comparison to the state of the art}

In contrast to comparable work in multiple objective optimization of energy systems with a high share of renewable energies, the selection of the appropriate number of typical periods is not based on Key Performance Indicator (KPI) from the machine learning algorithm in this procedure unlike [5, 6, 11, 12]. It is furthermore evaluated on the performance of the model of the energy system itself. The proposed procedure is similar to the method developed by Bahl et al. [8] and further refined in [9]. Two main distinctions are elaborated that make the method simpler and computationally more efficient:

1. Usage of two convergence criteria: This framework proposes two separate convergence criteria with the benefit of skipping the calculation of the computationally intensive second stage, the operating problem on the full time series, for most iterations.

2. Inclusion of unit decision: The second part of the framework includes the consideration of the unit decisions in the process of TSA evaluation, to either confirm convergence of unit decisions or compare possible solutions based on integer variables. 
3. The inclusion of the unit decisions makes it possible to use the TSA on a Multi-Objective Optimization (MOO) problem.

\subsection{Energy system model}

The data reduction technique is applied on a MILP approach of the optimal design and scheduling of a building energy system. The type and the size of the different components are the main decision variables in order to satisfy space heating, electricity and domestic hot water demand. There are nine technology options to choose from for each building: a natural gas boiler, an air water heat pump, a Solid Oxide Fuel Cell (SOFC), which serves as a cogeneration unit, two electrical heaters and two thermal storage tanks, one each for domestic hot water and space heating demand, a lithium ion battery and Photovoltaic (PV) panels. The building is connected to the fresh water, the natural gas and the electrical power grid.

The space heating demand is impacted by the conductive heat losses through the envelope, the heat capacity of the building, internal heat gains from appliances and people, as well es external heat gains from solar irradiation. The building mass can serve as thermal storage and the indoor temperature is variable within a preferred temperature range. This enables the option of heating the building at times of surplus energy, hence this approach is enabling optimal inclusion of renewable energy. In contrast, the domestic hot water is served at a fixed supply temperature of $60^{\circ} \mathrm{C}$ to respect hygienic standards [13]. The electricity demand of the building is modelled according to Swiss standard norms [13].

Energy balances of the system close the first law of thermodynamics whereas a discretized heat cascade ensures to respect the second law of thermodynamics. Cyclic constraints reset the indoor temperature and all thermal and electrical streams to its initial status at the end of each period to ensure that no energy is accumulated between different typical periods. The reader is transferred to [6] for further insights about the modeling approach. In the following, the main objective functions of the MILP problem are summarized before an overview of the case study and connected input data is provided.

\subsubsection{Objectives}

The MILP problem is defined as minimizing connected expenses. This includes the contribution of Operational Expenses (OPEX) and Capital Expenses (CAPEX), which are in general conflicting. Thus, a MOO approach is required. The $\mathrm{MOO}$ is implemented using the $\epsilon$-constraint method, which takes OPEX as main objective and solves the problem with increasing limits on acceptable CAPEX. The same principle is then repeated where both objectives invert the position.

The annual OPEX account for the interaction with the local power and gas grid (Equation 11. $c^{e l,+}, c^{e l,-}$ and $c^{n g,+}$ express purchase and feed-in tariffs, which are time independent in this study. $\dot{H}_{b, p, t}^{g r,+}$ represents the energy flow of natural gas purchased from the grid for building $b$ at time step $t$ and typical period $p$; similarly, $\dot{E}_{b, p, t}^{g r,+}$ and $\dot{E}_{b, p, t}^{g r,-}$ represent the electricity exchange with the grid. Annual values are integrated over each typical period $p$ and accounted with their frequency $d$.

$$
\boldsymbol{C}^{o p}=\sum_{b \in \mathbf{B}} \sum_{p \in \mathbf{P}} \sum_{t \in \mathbf{T}}\left(c^{e l,+} \cdot \dot{\boldsymbol{E}}_{\boldsymbol{b}, \boldsymbol{p}, \boldsymbol{t}}^{g r,+}-c^{e l,-} \cdot \dot{\boldsymbol{E}}_{\boldsymbol{b}, \boldsymbol{p}, \boldsymbol{t}}^{g r,-}+c^{n g,+} \cdot \dot{\boldsymbol{H}}_{\boldsymbol{b}, \boldsymbol{p}, \boldsymbol{t}}^{g r,+}\right) \cdot d_{t} \cdot d_{p}
$$

The annual CAPEX consist of the investment and replacement cost (Equation 2a), which are annualized with the interest rate $i$ and the duration $n$ of project [14]. The investment costs are further described in Equation $2 \mathrm{~b}$, where the parameters $i^{c 1}, i^{c 2}$ and the baremodule $b_{u}$ describe the linear version of the cost function. The binary variable $y_{b, u}$ represents the decision to install a unit $u$ in building $b$, whereas the continuous variable $f_{u}$ represents the unit size. If the lifetime of a unit $I_{u}$ exceeds the project horizon, it needs to be replaced (Equation 2C). In this study, only lithium batteries and cogeneration units need replacement.

$$
\begin{aligned}
& \boldsymbol{C}^{c a p}=\sum_{b \in \boldsymbol{B}} \frac{i(1+i)}{(1+i)^{n}-1}\left(\boldsymbol{C}_{b}^{i n v}+\boldsymbol{C}_{b}^{r e p}\right) \\
& \boldsymbol{C}_{b}^{i n v}=\sum_{u \in \boldsymbol{U}} b_{b, u} \cdot\left(i_{b, u}^{c 1} \cdot \boldsymbol{y}_{\boldsymbol{b}, \boldsymbol{u}}+i_{b, u}^{c 2} \cdot \boldsymbol{f}_{\boldsymbol{b}, \boldsymbol{u}}\right) \quad \forall b \in \mathbf{B} \\
& \boldsymbol{C}_{b}^{\text {rep }}=\sum_{u \in \boldsymbol{U}} \sum_{r \in \boldsymbol{R}} \frac{1}{(1+i)^{r \cdot l_{u}}} \cdot\left(i_{b, u}^{c 1} \cdot \boldsymbol{y}_{b, u}+i_{b, u}^{c 2} \cdot \boldsymbol{f}_{b, u}\right) \quad \forall b \in \mathbf{B}
\end{aligned}
$$

The combination of CAPEX and OPEX leads to the Total Expenses (TOTEX) of the project (Equation 3):

$$
C^{\text {tot }}=C^{o p}+C^{c a p}
$$

\subsubsection{Case study}

Two case studies with different levels of complexity demonstrate the proposed procedure. The first case study is a typical Swiss building located in the climatic zone of Geneva. The building is a residential, single- family 
home with 2 floors and in total $250 \mathrm{~m} 2$ heated surface from around 1950. Potential installation of $\mathrm{PV}$ Panels are assumed to be horizontally. On a full time series of one year, this case study leads to over 830 thousand constrains and 780 thousand variables, among which are almost 9000 binaries.

The second case study includes the very same building plus the 4 neighboring buildings, all residential buildings, among which one multi family home and 3 additional single family homes built between 1950-2000 . Additionally, all allocated roof surfaces are considered for possible $\mathrm{PV}$ panel installations, leading to 24 different roofs and their orientations to choose from. The modelling approach of oriented PV panels is reported in [15]. The second case study on a full time series of one year sums up to over 4.1 million constrains, almost 4 million variables among which are almost 44000 binaries.

Building specific parameters such as the heat transfer coefficient, the heat capacity or the supply and return temperatures of the heating system are derived from [16]. Typical annual values of the global irradiation and the external temperature are reported at [17]. The k-medoids clustering with the $\mathrm{R}$ package wcKMedoids is performed for aggregating one typical year. Furthermore, one extreme cold weather period is added, consisting of one single time-step.

The problem is formulated in AMPL Version 20191001 and solved with CPLEX 12.9.0.0 on a local machine with following processor details: Intel(R) Core(TM) i7-8559U CPU @ 2.70GHz. The relative tolerance between relaxed linear problem and best integer solution is set to mipgap=1e-8. The remaining CPLEX settings are equal to the default settings reported at [18].

\section{Results}

In a preprocessing step, Global Irradiation (GHI) and the external temperature (T) are clustered to different number of k-medoids. The length of one typical period is chosen to be $24 \mathrm{~h}$. Figure 3 displays the quality of the aggregation. The common procedure for selecting amount of typical periods is to define a slope threshold of the KPIs. A slope threshold of $10 \%$ would lead to around 10 typical periods.

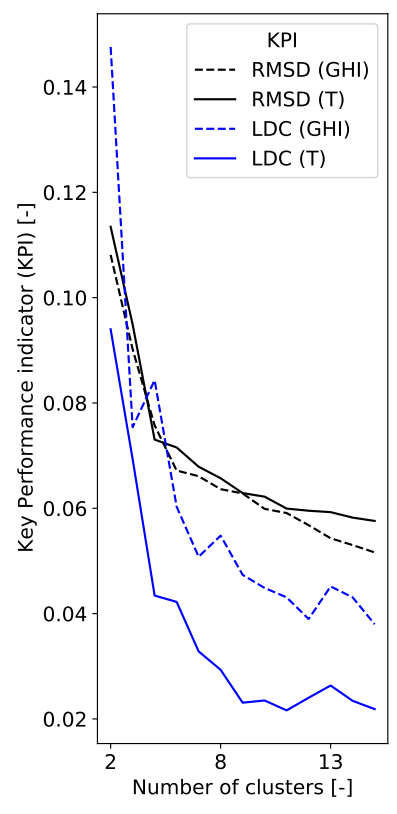

(a)

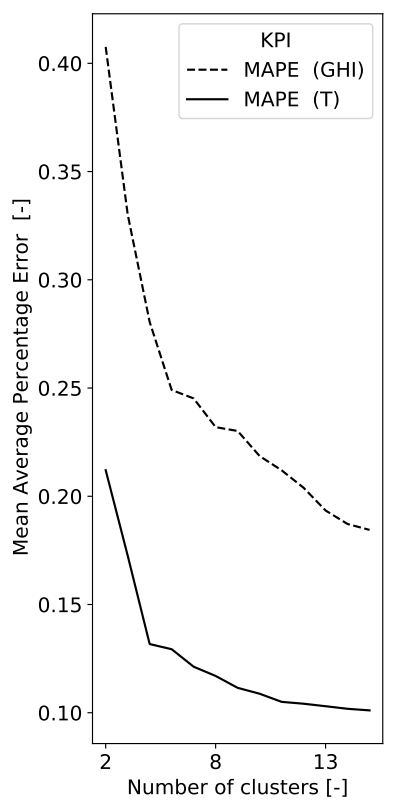

(b)

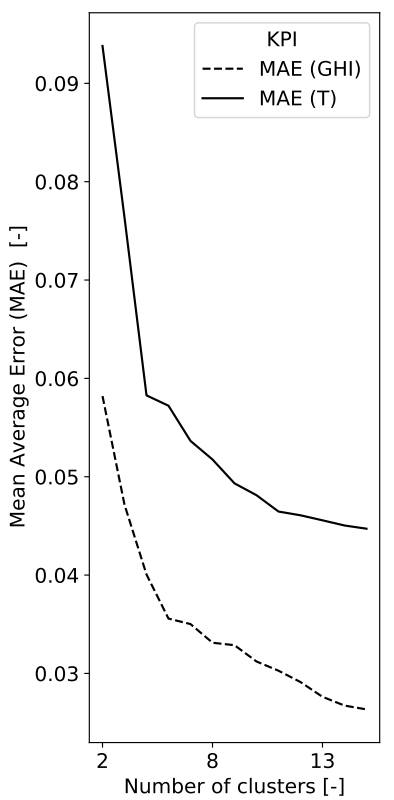

(c)

Figure 3: Key performance indicators (KPIs) of the k-medoids clustering of global irradiation (GHI) and the external temperature (T). a) Root mean square deviation (RMSD) and difference on the load duration curve (LDC) b) Mean average percentage error (MAPE) C) Mean average error (MAE).

\subsection{Algorithm part 1}

First, the AP with an increasing amount of clusters is solved. The objective function TOTEX which is equally weighting the two conflicting objectives of the MOO. Figure 4 demonstrates part one of the proposed algorithm for case study one, a typical residential building. The algorithm starts with two clusters. To be able to observe the relative change of the objective function, the $\mathrm{AP}$ is immediately solved with 3 typical periods. Already after these 3 clusters the convergence criteria $\epsilon_{a}$ is approximately $5 \%$. The unit decisions are fixed and the full time series is solved. Similar to the criteria $\epsilon_{a}, \epsilon_{b}$ is below $5 \%$ and therefore 3 typical periods are chosen as number of typical clusters for further investigations in the second part of the algorithm. For demonstration purposes, the aggregated and the operating problem on the full time series is solved up to 12 typical days. After 7 typical 
days, the difference of the objective functions, and therefore the second convergence criteria stays even below $1 \%$.

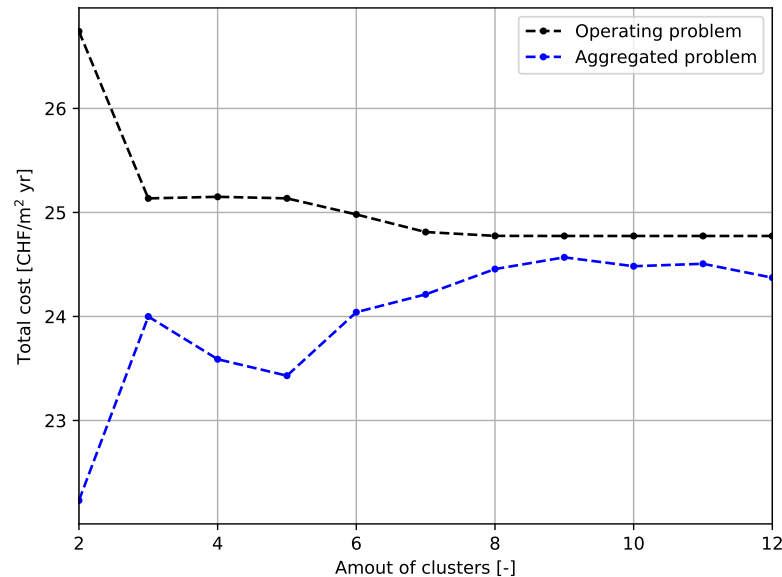

(a)

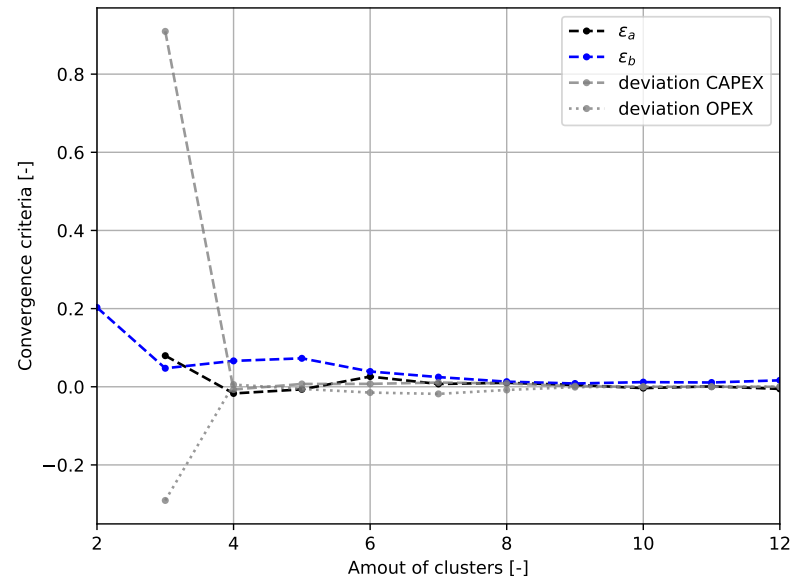

(b)

Figure 4: Demonstration of part 1 of the algorithm, case study 1 Building with objective TOTEX Impact of different number of clusters on a) the objective function b) the convergence criteria $\epsilon_{a}$ and $\epsilon_{b}$, as well as deviation of CAPEX and OPEX to $n-1$ clusters.

Figure 5 visualizes the algorithm of the second and more complex case study. At three clusters the first convergence criteria $\epsilon_{a}$ is over $30 \%$ and not satisfactory. Thus, one additional cluster is added for which $\epsilon_{a}$ is reaching $5 \%$. Hence, the $\mathrm{OP}$ is solved which leads to $\epsilon_{b}=4 \%$. For the second case study 4 typical days seems appropriate for executing the second part of the algorithm.

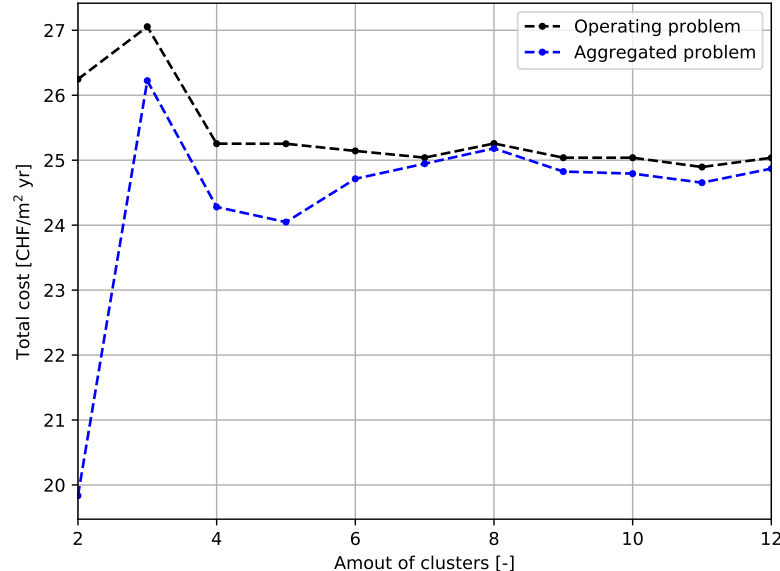

(a)

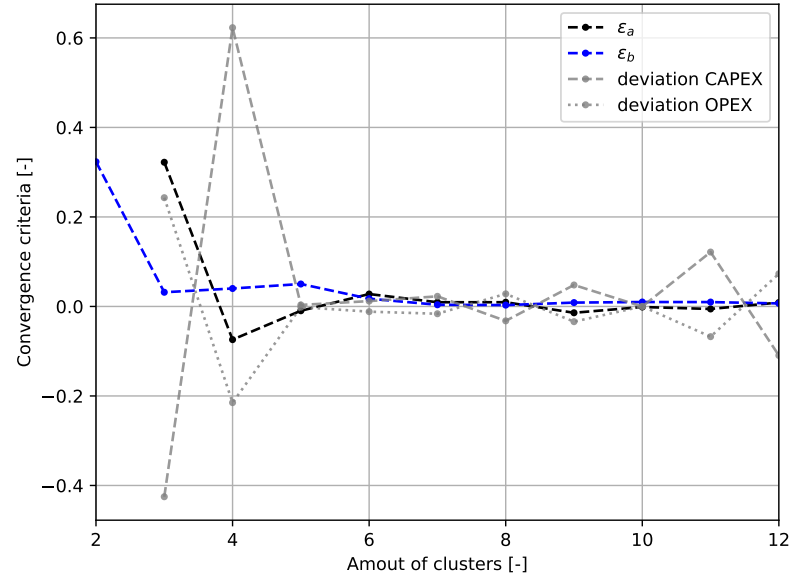

(b)

Figure 5: Demonstration of part 1 of the algorithm, case study 5 Buildings with objective TOTEX Impact of different number of clusters on a) the objective function b) the convergence criteria $\epsilon_{a}$ and $\epsilon_{b}$, as well as deviation of CAPEX and OPEX to $n-1$ clusters.

\subsection{Algorithm part 2}

The second part of the algorithm aims at looking at the integer decisions which are chosen for the AP. For demonstration purposes not only integer decisions are visualised but as the objective is TOTEX. Figure 6additionally shows the annual energy which is exchanged with the network and contributes to the objective. For two clusters an airwater heatpump in combination with electrical heater and thermal storage tanks seems to be the most economical decision. For three clusters, PV modules are additionally installed. This configuration stays the same for all further investigated number of clusters. Therefore, the three typical periods can be confirmed and further used during $\mathrm{MOO}$ 

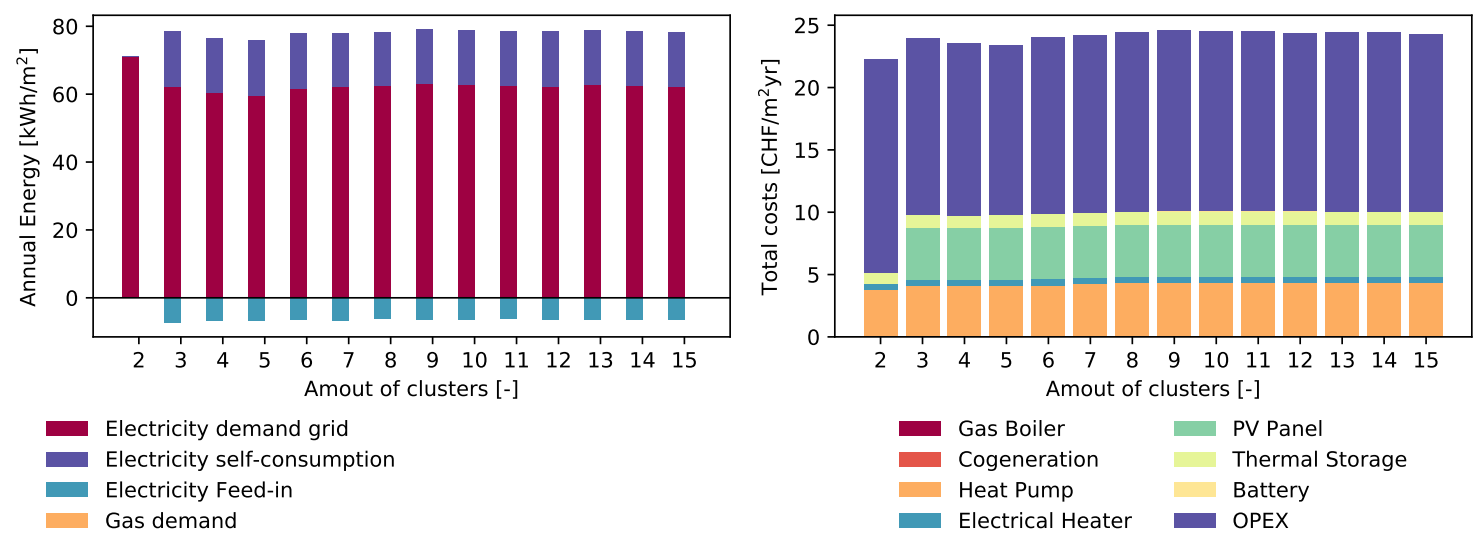

Figure 6: Overview of unit decision and network operation for the aggregated problem (AP) with different amount of typical periods for minimizing TOTEX Case study with 1 residential building.

Figure 7 visualizes part two of the algorithm for the more complex case study of 5 residential buildings with individual roof orientation. For the suggested amount of four clusters, the unit decisions are air water heat pumps, additional electrical heaters for peak loads, thermal storage tanks and $\mathrm{PV} \mid$ panels. These decisions stay the same for the observation period of $m=3$ and the TSA can be ended with 4 typical periods for the second case study.
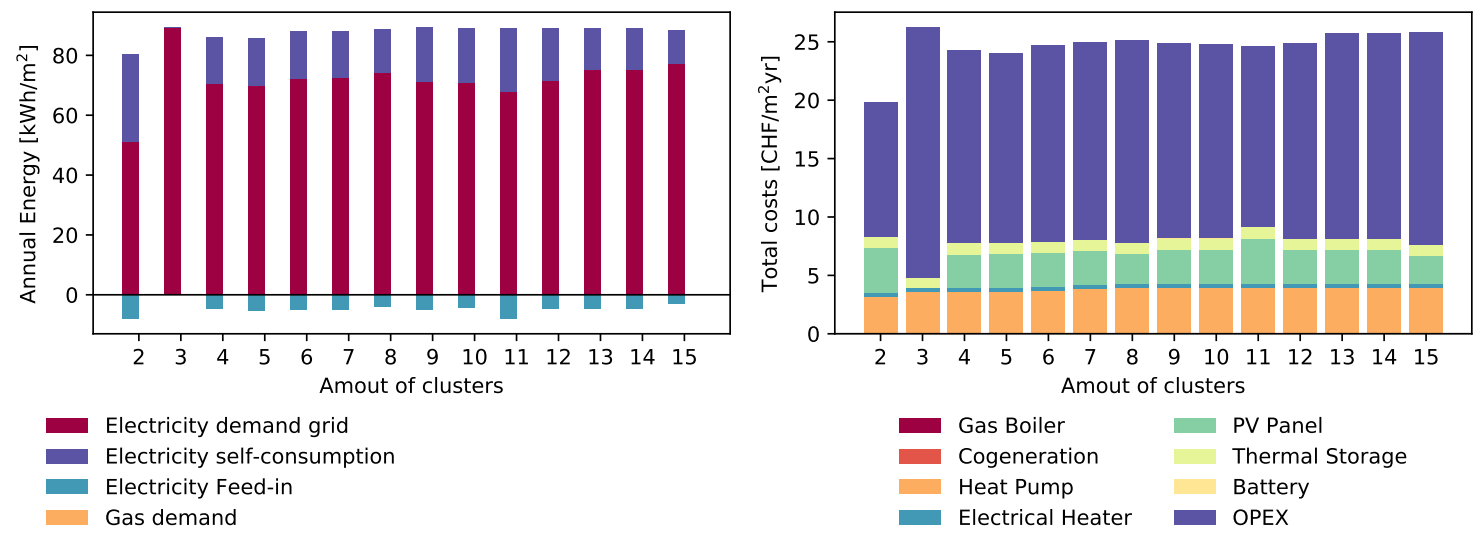

Figure 7: Overview of unit decision and network operation for the aggregated problem (AP) with different amount of typical periods for minimizing TOTEX Case study of a network consisting of 5 residential building with individual|PV|orientation.

\subsection{Multi-criteria decision making}

The overall goal is to propose a TSA method which is usable not for only a single run of one objective, but which can be used for all further investigations in the $\mathrm{MOO}$ domain. Therefore, this section is presenting the performance of the demonstrated TSA in context with the MOO of case study 1. Figure 8a compares the Pareto curves of the $\mathrm{AP}$ to the associated $\mathrm{OP}$ on the full time series for the previously resulting 3 typical periods. Related unit decisions of each Pareto - optimal scenario are displayed in Figure 9. The CAPEX ranges from approximately $3 \mathrm{CHF} / \mathrm{m}^{2}$ heated surface (Scenario 1 ) to almost $30 \mathrm{CHF} / \mathrm{m}^{2}$ (Scenario 8). The unit decision of Scenario 1 is based on natural gas, only using a gas boiler and small thermal storage tanks. For this Scenario, the $\mathrm{AP}$ and its linked $\mathrm{OP}$ is almost identical on the Pareto frontier (Figure $8 \mathrm{a}$ ). In contrast, the unit decision for Scenario 8 is more diverse and includes renewable energy sources such as PV panels and an air water- heat pump but also thermal and electrical storage (Figure 9). The performance of these units are all depending on the weather data or, in terms of the storage, the optimal scheduling within one typical period. Nevertheless, comparing the OPEX of the AP and the linked OP shows a deviation by only $3 \%$. 


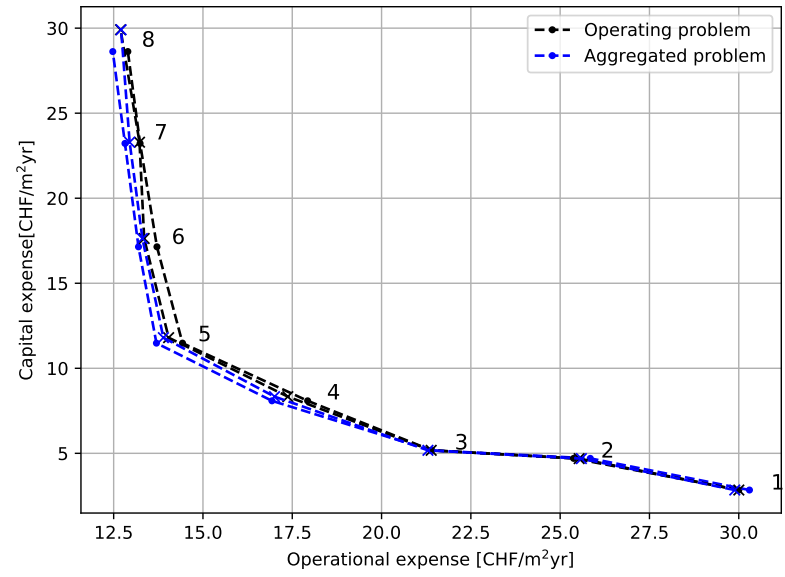

(a)

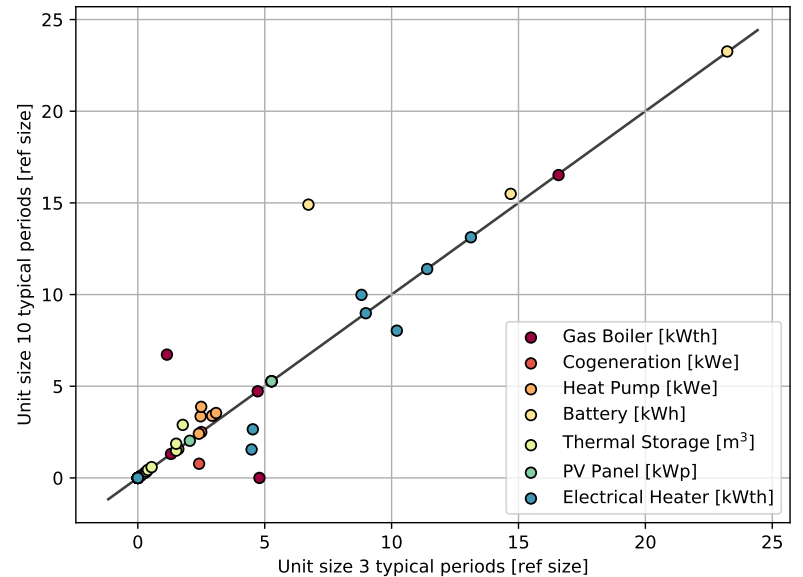

(b)

Figure 8: a) CAPEX-OPEX Pareto frontier for case study 1. Aggregated problem (AP) with 10 typical periods (x) and 3 typical periods $(\bullet)$. b) Direct comparison of 9 unit choices in each of the 8 Pareto scenarios with 3 and 10 typical periods.
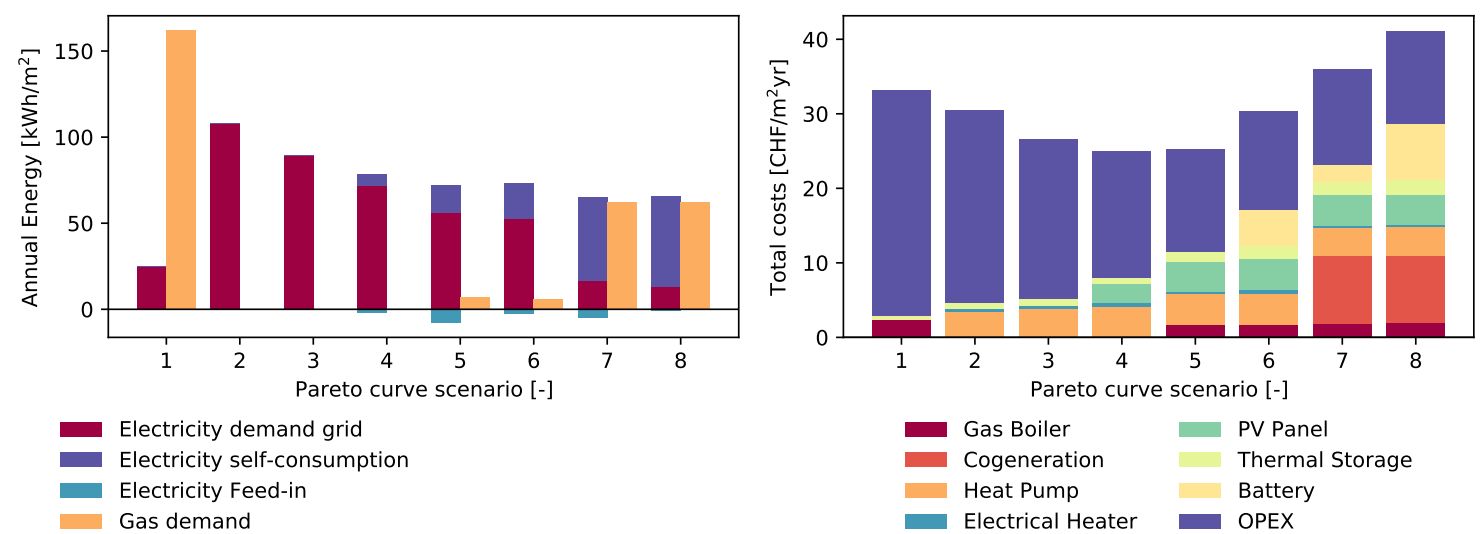

Figure 9: Overview of different unit decisions along the Pareto optimal scenarios. Case study 1 with 3 typical periods.

\subsection{Comparison to the state of the art}

The proposed approach leads to a significantly lower amount of required typical periods compared to the state of the art procedure, which is based on the selection on $\mid \mathrm{KPl} s$ of the machine learning algorithm itself. Using k-means aggregation method on the annual heating demand, [5] identifies 13 typical days, whereas k-medoids clustering of the weather data reveals 8 typical days for [6]. For the addressed case study, conventional approaches would result in approximately 10 typical days. Therefore, this solution is compared to the 3 typical days obtained from the proposed TSA algorithm. Figure $8 \mathrm{a}$ is visualizing the CAPEX- OPEX Pareto front of case study 1 for 3 and 10 typical periods. Both Pareto curves of the AP are basically identical for lower scenarios and divert less than $2 \%$ for higher investment scenarios. Similar observations are possible for the associated $\mathrm{OP} s$. This shows that 3 typical typical periods are enough in terms of cost calculation during $\mathrm{MOO}$ Figure $8 \mathrm{~b}$ compares the unit sizes, which are chosen at each pareto optimal scenario. Nine unit sizes in eight Pareto points are directly compared, although different epsilon constraints are identified in the process of both $\mathrm{MOO}$. It is apparent that most of the 72 unit decisions are identical, even among different scenarios. The size of $\mathrm{PV}$ panels in all 8 Scenarios are identical, similar conclusion is valid for electrical heaters and storage tanks for space heating. It seems that for higher investment scenarios and a larger number of typical periods, back-up units for domestic hot water and the batteries are reduced while base-load equipment such as heat pumps are slightly increasing size. However, for a clean picture, this comparison has to be done comparing same epsilon constraints for the two MOO approaches.

Figure 10 illustrates the computational benefit of the proposed approach. Applying the method proposed by [9], the solution of the AP took seconds or up to a few minutes, whereas the solution of OP consistently took about four times longer. Thus, the proposed framework reaches lower total computational times due to a simple 


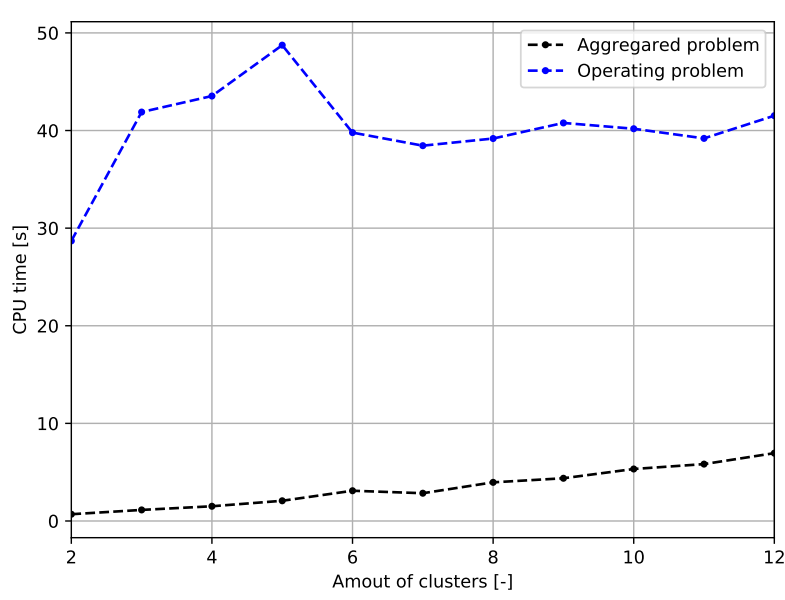

(a)

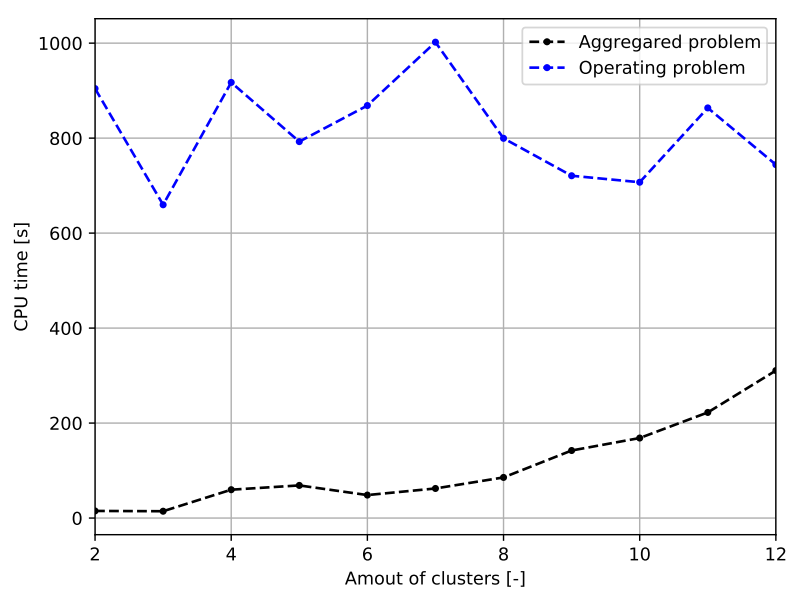

(b)

Figure 10: Computation time of aggregated time series including unit decisions (black) and the operation problem of the full time series, without unit decision (blue) a) case study of one building b) case study of 5 buildings.

elimination of time consuming computing steps. Table 1 summarizes the CPU time for the TSA as well as for the application during the presented $\mathrm{MOO}$ and compares it to the respective state of the art. The proposed method for TSA is reducing the CPU time by $40 \%$ and even for $61 \%$ for the more complex case study 2 . This demonstrates the benefit of the proposed method especially for complex energy systems. The reduction of the required typical periods is leading to a decrease in CPU time of around $90 \%$ for the presented MOO

Table 1: Comparison of the proposed approach to the state of the art.

\begin{tabular}{|c|c|c|c|c|c|}
\hline & \multicolumn{2}{|l|}{ Case study 1} & \multicolumn{2}{|l|}{ Case study 2} \\
\hline & & CPU time [s] & Comparison[\%] & CPU time [s] & Comparison[\%] \\
\hline TSA & State of the art [19] & 72 & 100 & 2570 & 100 \\
\hline & Proposed approach & 43 & 60 & 1000 & 39 \\
\hline $\mathrm{MOO}$ & State of the art [6] & 96 & 100 & 2870 & 100 \\
\hline & Proposed approach & 9 & 9 & 380 & 13 \\
\hline
\end{tabular}

\section{Conclusion}

This work analyzes the impact of data reduction techniques on complex energy systems. Energy systems which include a high share of renewable energies, require a time resolution of a few hours but planning and designing them needs the consideration of several decades. Literature in the field addresses this issue by using machine learning techniques to identify typical periods and thus reduce the input space. The presented work is combining a k-medoids clustering approach with systematic performance evaluation of the MILP framework of the energy system. Compared to the state of the art, this approach includes the consideration of the integer decision of the installed technologies in the energy system. Furthermore the CPU time of the TSA is reduced by more than $40 \%$ by substituting computational intensive steps with an additional convergence criteria. The most interesting results however, are related to the application on MOO. The proposed method leads to a remarkable reduction in typical periods compared to the selection technique of the state of art in $\mathrm{MOO}$. This reduces the CPU time by $90 \%$, while the pareto curves are deviating by up to $2-3 \%$ in the presented case. The integer decisions are identical along the curve. Unit sizes require a more detailed investigation to lead to meaningful results, which could further be extended to the inclusion of seasonal storage. One possible extension of this work is the consideration of not one typical year but the full project horizon for the TSA, which brings along the challenge of weather prediction subjected to climate change. Furthermore, the impact of extreme conditions could be addressed by parallel detection of outliers in the aggregation method.

\section{Acronyms}

AP Aggregated Problem; CAPEX Capital Expenses; GHI Global Irradiation; KPI Key Performance Indicator; MAPE Mean Average Percentage Error; MILP Mixed-Integer Linear Programming; MOO Multi-Objective Optimization; OP Operating Problem; OPEX Operational Expenses; PV Photovoltaic; RMSD Root Mean Square Deviation; SOFC Solid Oxide Fuel Cell; SSE Sum of Squared Errors; TOTEX Total Expenses; TSA Time 


\section{Nomenclature}

\section{Parameter}

$\epsilon_{a} \quad$ First convergence criteria

$\epsilon_{b} \quad$ Second convergence criteria

$b \quad$ Baremodule

c Energy tariff

d Duration

i Interest rate

$i^{c 1} \quad$ Fixed Investment Cost

$i^{c 2}$ Continuous Investment Cost

I Lifetime

$n$ Project horizon

Variables

C Cost

E Electricity

$\boldsymbol{f} \quad$ Sizing variable

$\boldsymbol{H} \quad$ Natural gas

$\boldsymbol{y}$ Decision variable, binary

$\begin{array}{rll} & \text { Sets } & \\ - & \mathrm{B} & \text { Building } \\ - & \mathrm{P} & \text { Period } \\ - & \mathrm{R} & \text { Replacement } \\ \mathrm{CHF} / \mathrm{kWh} & \mathrm{T} & \text { Timestep } \\ \mathrm{h} & \mathrm{U} & \text { Utility } \\ - & \text { Superscripts } \\ \mathrm{CHF} & + & \text { Supply } \\ \mathrm{CHF} / \diamond & - & \text { Demand } \\ \mathrm{yr} & \text { cap } & \text { Capital } \\ \mathrm{yr} & \text { el } & \text { Electricity } \\ & g r & \text { Grid } \\ \mathrm{CHF}(/ \mathrm{yr}) & \text { inv } & \text { Investment } \\ \mathrm{kW}(\mathrm{h}) & n g & \text { Natural Gas } \\ \diamond & \text { op } & \text { Operation } \\ \mathrm{kW} & \text { rep } & \text { Replacement } \\ {[-]} & \text { tot } & \text { Total }\end{array}$

b

p

$r$

$\mathrm{t}$

u

\section{References}

[1] T. Stocker, Climate change 2013: the physical science basis: Working Group I contribution to the Fifth assessment report of the Intergovernmental Panel on Climate Change. Cambridge university press, 2014.

[2] M. Hoffmann, L. Kotzur, D. Stolten, and M. Robinius, "A Review on Time Series Aggregation Methods for Energy System Models," Energies, vol. 13, p. 641, Feb. 2020.

[3] T. Schütz, M. H. Schraven, M. Fuchs, P. Remmen, and D. Müller, "Comparison of clustering algorithms for the selection of typical demand days for energy system synthesis," Renewable Energy, vol. 129, pp. 570$582,2018$.

[4] L. Kotzur, P. Markewitz, M. Robinius, and D. Stolten, "Impact of different time series aggregation methods on optimal energy system design," Renewable Energy, vol. 117, pp. 474-487, Mar. 2018.

[5] S. Fazlollahi, S. L. Bungener, P. Mandel, G. Becker, and F. Maréchal, "Multi-objectives, multi-period optimization of district energy systems: I. Selection of typical operating periods," Computers \& Chemical Engineering, vol. 65, pp. 54-66, June 2014.

[6] P. M. Stadler, Model-based sizing of building energy systems with renewable sources. PhD thesis, EPFL, 2019.

[7] P. G. Brodrick, C. A. Kang, A. R. Brandt, and L. J. Durlofsky, "Optimization of carbon-capture-enabled coal-gas-solar power generation," Energy, vol. 79, pp. 149-162, 2015.

[8] B. Bahl, A. Kümpel, H. Seele, M. Lampe, and A. Bardow, "Time-series aggregation for synthesis problems by bounding error in the objective function," Energy, vol. 135, pp. 900-912, Sept. 2017.

[9] N. Baumgärtner, B. Bahl, M. Hennen, and A. Bardow, "RiSES3: Rigorous Synthesis of Energy Supply and Storage Systems via time-series relaxation and aggregation," Computers \& Chemical Engineering, vol. 127, pp. 127-139, 2019.

[10] P. Gabrielli, M. Gazzani, E. Martelli, and M. Mazzotti, "Optimal design of multi-energy systems with seasonal storage," Applied Energy, vol. 219, pp. 408-424, 2018.

[11] T. Schütz, X. Hu, M. Fuchs, and D. Müller, "Optimal design of decentralized energy conversion systems for smart microgrids using decomposition methods," Energy, vol. 156, pp. 250-263, Aug. 2018.

[12] F. Domínguez-Muñoz, J. M. Cejudo-López, A. Carrillo-Andrés, and M. Gallardo-Salazar, "Selection of typical demand days for CHP optimization," Energy and Buildings, vol. 43, pp. 3036-3043, Nov. 2011.

[13] SIA, "2024:2025 Raumnutzungsdaten für die Energie- und Gebäudetechnik," Schweizerischer Ingenieur und Architektenverein, 2015. 
[14] R. Turton, ed., Analysis, synthesis, and design of chemical processes. Upper Saddle River, NJ: Prentice Hall, 4th ed ed., 2012.

[15] L. Middelhauve, F. Baldi, P. Stadler, and F. Maréchal, "Grid-Aware Layout of Photovoltaic Panels in Sustainable Building Energy Systems," Frontiers in Energy Research, vol. 8, p. 573290, Feb. 2021.

[16] L. Girardin, A GIS-based Methodology for the Evaluation of Integrated Energy Systems in Urban Area. PhD thesis, EPFL, 2012.

[17] DOE, "Weather Data | EnergyPlus, U.S. Department of Energy's (DOE) Building Technologies Office (BTO) and National Renewable Energy Laboratory (NREL).," Mar. 2020. https://energyplus.net/weather.

[18] "CPLEX Options for AMPL," Feb. 2020. https://ampl.com/products/solvers/solvers-we-sell/cplex/options/.

[19] B. Bahl, Optimization-Based Synthesis of Large-Scale Energy Systems by Time-Series Aggregation; 1. Auflage, vol. RWTH Aachen University. RWTH Aachen University, 2018. Artwork Size: pages 1 OnlineRessource (XXI, 148 Seiten) : Illustrationen Pages: pages 1 Online-Ressource (XXI, 148 Seiten) : Illustrationen Publication Title: Dissertation. 